\title{
Repurposing Benzbromarone as Antifolate to Develop Novel Antifungal Therapy for Candida Albicans
}

Somdutt Mujwar ( $\sim$ somduttmujwar@gmail.com )

GLA University https://orcid.org/0000-0003-4037-5475

Avanish Tripathi

GLA University Institute of Pharmaceutical Research

\section{Research Article}

Keywords: Benzbromarone, Antifungal, Candida albicans, DHFR, Repurposing, White-fungus, COVID-19.

Posted Date: November 23rd, 2021

DOI: https://doi.org/10.21203/rs.3.rs-1057044/v1

License: (c) (1) This work is licensed under a Creative Commons Attribution 4.0 International License.

Read Full License 


\section{Abstract}

Fungal infections in humans are responsible for mild to severe infections resulting in the systemic effects responsible for a large amount of mortality. The invasive fungal infections are having similar symptomatic effects to those of COVID-19. The COVID-19 patients are immunocompromised in nature and have a high probability of developing severe fungal infections resulting in the development of further complications. The existing antifungal therapy is having associated problems related to the development of drug resistance, sub-potent in nature, and the presence of undesirable toxic effects. The fungal dihydrofolate reductase is an essential enzyme involved in the absorption of dietary folic acid and its conversion into tetrahydrofolate, which is a coenzyme required for the biosynthesis of the fungal nucleotides. Thus, in the current study, an attempt has been made to identify potential folate inhibitors of Candida albicans by a computational drug repurposing approach. Benzbromarone is identified as a potential anti-folate agent based upon the molecular docking simulation-based virtual screening followed by the molecular dynamic simulation of the macromolecular complex for the development of a novel therapy for the treatment of candidiasis.

\section{Introduction}

Fungal infection in humans ranges from mild skin disease-causing rashes and itching to fungal pneumonia, meningitis, and bloodstream infections responsible for causing a large number of fatalities. [1] The fungal infection responsible for systemic effects is considered Invasive Fungal Infections (IFI). Some of the common IFI diseases, like coccidioidomycosis, blastomycosis, histoplasmosis, etc., have symptoms like cough, cold, fever, and breathing difficulties, similar to that of COVID-19. The fungi responsible for causing such types of symptomatic effects are highly communicable to humans through air. IFI pneumonia is one of the probable causes of respiratory illness in COVID-19 negative patients. [2]

Patients suffering from COVID-19 infections are supposed to have compromised immunity and are also at a high risk of healthcare-associated infections like candidemia and other systemic fungal infections caused by Candida. [3, 4] Candidemia and other fungal pneumonias can also show synergistic effects with the SARS-CoV-2 virus, making the infection more complicated and responsible for increasing the mortality rate. It has been reported that the fungal co-infections in the COVID-19 patients are showing resistance to the existing antifungal therapy. [5]

Azoles are the most widely used antifungal drugs inhibiting pathogenic ergosterol biosynthesis. They nowadays have limited clinical utility because of the development of pathogenic resistance. The polyene compound amphotericin-B has broad spectrum antifungal activity through the disruption of the fungal membrane by interacting with ergosterol, but its systemic use has serious toxic effects, limiting its medicinal utility.[6] Echinocandins is a recently approved antifungal agent effective only against the Candida species by targeting their cell wall biosynthesis via intravenous administrations. $[7,8]$ 
It is a matter of high concern that the mediocre efficacy and limited clinical utility of the available three major classes of antifungal agents is responsible for increasing mortality rates in patients having IFI with a high rate of transmission of the infection among humans. More than one-third of the patients with Candida infections resisted the therapy with azoles like fluconazole, voriconazole, as well as isavuconazole. [9] The desired therapeutic effects were also not achieved by using echinocandin and amphotericin-B against candidiasis.

Therefore, to counter the resisted fungal pathogen, there is an urgent requirement to develop a novel antifungal agent with amended therapeutic efficacy, patient safety, and broad-spectrum of activity to counter the development of drug resistance by the fungal pathogen against the existing antifungal therapy and control the complications caused by fungal co-infection in COVID-19 patients.

Tetrahydrofolate is a vital coenzyme acting as a carrier for the transfer of a carbon unit for various cellular enzymes during their interconversion between various oxidative states required for the biosynthesis of important metabolites like methionine and purines. In the absence of necessary enzymes, mammals are incompetent for the biosynthesis of folate and depend only upon the dietary source in the form of folic acid. Cellular uptake of folic acid is regulated through a specialized transport mechanism followed by its conversion by dihydrofolate reductase (DHFR) to activated tetrahydro folate (THF). While the prokaryotes and the majority of the microbial eukaryotes lack this mammalian transport system required for the cellular uptake of folic acid. Thus, they have to synthesize the de novo folic acid by themselves to satisfy their biological needs. Targeting the folate biosynthetic pathway of pathogenic microbes by developing a suitable antifolate is a highly successful approach in developing different types of antimicrobial therapies for humans. $[7,10]$

Drug repurposing is a trending approach to identification of a newer pharmacological role for an existing approved drug, whereas establishing the newer mechanism of an existing approved drug by identifying its potent binding affinity towards an alternate drug target is called drug repositioning. The high cost as well as the slow pace involved in the traditional drug development process are becoming a barrier in the combat against the new diseases affecting mankind. $[11,12]$ Thus, computational repurposing and repositioning of existing approved drugs is an emerging trend for developing newer therapies to counter upcoming human diseases. Drug repurposing is the preferred approach for developing novel therapies because it saves a lot of time as only the binding interactions-based affinity of the molecule against the specific macromolecular drug target has to be established as the existing approved drugs have an established pharmacokinetics and toxicity profile. Data-driven computational approaches applied to the repurposing of existing drugs is a low budget approach having a higher rate of success in a short span of developmental time. $[13,14,11]$

Thus, in the current study, we are trying to develop reposition the existing FDA approved drugs against the DHFR of $C$. albicans by using molecular docking simulation based computational repurposing molecular dynamics for their further validation. 


\section{Material And Methodology}

The computational drug repurposing of existing approved drugs against the DHFR enzyme of C. albicans was performed by using a computer system having 10th generation i7 processor with 16GB of randomaccess memory and 4GB of graphic memory card wth the help of various molecular modeling tools including AutoDock, Desmond, PyMol, Chimera, etc.

The molecular docking simulation based virtual screening of a ligand library consisting of 2880 FDA approved drugs against the fungal DHFR enzyme of $C$.albicans was performed by underneath procedures.

\subsection{Molecular Docking Simulation}

The three-dimensional structure model of DHFR enzyme of $\mathrm{C}$. albicans complexed with NADPH and $\mathrm{e}$ meta-heterobiaryl propargyl-linked (18G) antifolate ligand was procured from the RCSB protein data bank (pdb id-4HOE). $[15,16]$ The complexed antifolate ligand $18 \mathrm{G}$ was separated from the macromolecular complex by using software Chimera.[17] The macromolecular target was prepared for molecular docking simulation by addition of polar hydrogens and Gasteiger charge to the amino acid residues with even distribution[12, 18, 14]. Ligand 18G was prepared for the docking simulation by assigning nonrotatable, rotatable and unrotatable bonds. The active ligand binding site of the fungal DHFR receptor was confirmed by observing the active binding interaction of the bioactive complexed ligand 18G by using Discovery Studio Visualizer.[19] Revealed binding site of the macromolecular target was further utilized for finalizing the size and position of the grid-box required to perform the docking simulations. The gridbox was prepared by centering the complexed bioactive ligand to cover its each and every extended conformation as well as the binding residues actively interacting with it.[13,20] The molecular docking simulation process for the fungal DHFR is validated by re-docking the separated bioactive ligand by considering the overlay and chemical resemblance of the docked conformation of the ligand with respect to its bioactive crystallized conformation.[21]

\subsection{Virtual screening of the Drug Library}

The validated docking parameters were further utilized for performing the in-silico screening of a ligand library consisting of 2880 FDA approved drug molecules against the fungal DHFR receptor.[22, 23] The virtual screening against the fungal DHFR receptor is performed with intent to identify potential leads having high affinity for the antifungal drug target.[24, 11, 12]

\subsection{Molecular Dynamic Simulation}

To analyses the binding stability and binding patterns of the potential hits obtained after virtual screening, molecular dynamic simulations for 10 ns was performed using the Desmond module of Schrödinger for the macromolecular complex of DHFR with all the shortlisted leads. $[25,26]$ Based on the stability of the ligands within the macromolecular complex observed in the dynamics simulation for shorter duration of $10 \mathrm{~ns}$, the dynamics simulation was amplified for $100 \mathrm{~ns}$ for the most stable ligand. 
An orthorhombic simulation box was prepared and a minimum distance of $10 \AA$ was set between the box wall and ligand-protein complex. A TIP3P explicit water model was used. System was neutralized by the addition of counter ions and isosmotic environment was made by providing $0.15 \mathrm{M} \mathrm{NaCl}$. 2000 iterations with convergence criteria of $1 \mathrm{kcal} / \mathrm{mol} / \AA ̊$ was used for system energy minimization. This energy minimized complex system was then subjected to $100 \mathrm{~ns}$ molecular dynamic simulations. A constant temperature of $300 \mathrm{~K}$ and a constant atmospheric pressure of 1.013 bars was maintained during simulation. An energy interval of $1.2 \mathrm{ps}$ and trajectory path was set at 9.6. At the end the trajectories were utilized to generate the simulation interaction diagrams.

\section{Results}

\subsection{Molecular Docking Simulation}

Macromolecular complex of fungal DHFR receptor DDR1 procured from the RCSB database protein data bank consisting two polypeptide chains of 192 amino acids. Chain A was retained to perform docking simulation while chain B was removed. The processed monomeric macromolecular structure has been represented in Fig. I. The ligand is having 16 aromatic carbons and all the six flexible bonds were kept rotatable in the current docking protocol. Bioactive complexed ligand $18 \mathrm{G}$ is having major interactions with Pro63, Ile62, Thr58, Ile112, Phe36, Ile9, Ala11, Ile33, and Glu32 amino acids within the active ligand binding site of the fungal DHFR receptor. An imaginary three-dimensional grid-box was prepared by wrapping all the macromolecular binding residues interacting with the ligand. The grid-box was shown in the Fig. II. and their coordinates were tabulated in Table I.

\section{Table I}

The grid coordinates for the fungal DHFR protein.

\begin{tabular}{|llllllll|}
\hline Proteins & $x-D$ & $y-D$ & z-D & Spacing $(\AA)$ & $x$ center & y center & z center \\
\hline $4 \mathrm{HOE}$ & 40 & 40 & 40 & 0.408 & 0.679 & 5.441 & 32.464 \\
\hline
\end{tabular}

\section{Table II}

Docking results of $18 \mathrm{G}$ ligand against the fungal DHFR protein.

\begin{tabular}{|llllll|}
\hline Proteins & Ligand & Interacting residues & RMSD & $\begin{array}{l}\text { Binding } \\
\text { energy } \\
\text { (kcal/mol) }\end{array}$ & $\begin{array}{c}\text { Binding } \\
\text { affinity } \\
\text { (nM) }\end{array}$ \\
\hline 4HOE & $18 \mathrm{G}$ & $\begin{array}{l}\text { Pro63, lle62, Thr58, lle112, Phe36, Ile9, } \\
\text { Ala11, Ile33, and Glu32 }\end{array}$ & 0.6 & -9.93 & 52.84 \\
\hline
\end{tabular}

3.2 Virtual screening of the Drug Library 
Out of ligand library containing 2880 FDA approved drugs, drug molecules having potential binding affinity for the fungal DHFR receptor were selected on the basis of the lowest binding energy in the predefined range of -5 to $-15 \mathrm{kcal} / \mathrm{mol}$. The binding energy obtained for top ten drug molecules after performing molecular docking simulation based virtual screening is given in Table III.

\section{Table III}

The binding energy of the top ten drug molecules obtained after Autodock based virtual screening of ligand library containing 2890 FDA approved drugs.

\begin{tabular}{|c|c|c|c|c|}
\hline S. No. & ZINC Id & Name of Compound & Structure & $\begin{array}{c}\text { Binding Energy } \\
(\mathrm{kcal} / \mathrm{mol})\end{array}$ \\
\hline 1 & ZINC03813024 & Pipotiazine & & -10.15 \\
\hline 2 & ZINC13444037 & Tasosartan & & -10.08 \\
\hline 3 & ZINC00608205 & Benzbromarone & & -10.07 \\
\hline 4 & ZINC01482077 & Gliquidone & & -10.07 \\
\hline 5 & ZINC03831128 & Mifepristone & & -9.99 \\
\hline
\end{tabular}

\subsection{Molecular Dynamic Simulation}

The benzbromarone was found to be most stabilized ligand after performing dynamic simulation for shorter duration of time and thus the same procedure has been amplified for a longer duration of $100 \mathrm{~ns}$ 
for identification of the stability of the complex ligand benzbromarone within the macromolecular target.

RMSD protocol measures the stability and conformational changes in the protein backbone during the simulation time. Based on MD results we revealed that, docked complex trajectories were found to be stable throughout the simulation with a mean RMSD value ranging from $4 \AA$ (Fig. IV) showing stable behavior with lesser fluctuation.

We also calculated the RMSF values by using the Ca atoms of enzymes from the stable trajectory to get insight the structural fluctuations of active amino acid residues in the enzyme. The lower the fluctuations the lesser in the mobility of amino acid in the active site, and vice versa. In RMSF plot the amino acid residues are shown in the $x$-axis and their RMSF value in $y$-axis. The active amino acid residues were evaluated and was observed to be show lesser RMSF value for the complex system. The average fluctuation range of the Benzbromarone was observed to be 1.2-1.8 , Thus, RMSF analysis of selected complexes showed comparable fluctuations in active sites residues that are important for molecular interactions.

The establishment of hydrogen bond, hydrophobic interaction and ionic interaction between the protein ligand complex during the MD simulation is responsible for their stability. Thus, we also evaluated the strength of these interaction to quantify the stability of our screened compounds against the DHFR enzymes. The interaction analysis was performed using the simulation interaction analysis module of Desmond in the maestro. The interaction analysis of Benzbromarone with DHFR reveals that hydroxy molecules interact with PHE-36 amino acid by hydrophobic interaction (56\%) and amino acid LYS-37 formed a cationic bridge (36\%) with benzenes nucleus (Fig. V).

\section{Discussion}

Fungal infections are a well-described phenomenon in critically ill, instinctively ventilated COVID-19 patients. In an earlier report, four fungal pathogens, namely Candida albicans, Candida glabrata, Aspergillus flavus and Aspergillus fumigatus, attributed central line sources for infection. The mortality rate of covid-19 patients was $38 \%$, but it increased drastically up to $51 \%$ in fungal species as copathogens. The life expectancy enhanced in patients receiving antifungal therapy suggested that the drug dose regimen is appropriate.[27, 28]

The available therapeutic for antifungals has a number of limitations. A particular strong antifungalresistance strain observed against Azole derivatives and amphotericin-B in all the formulations showed nephrotoxicities.[29] Literature reported that $15 \%$ of covid patients are also fungal infected; therefore, to meet this criterion, it is necessary to develop a new, significant, and effective antifungal drug with the least adverse effect, which is in contempt of a growing pool of resistant pathogenic organisms.[30, 31]

The folate metabolic pathway is vital for the generation of essential precursors for physiological metabolism for cells (prokaryotes and eukaryotes). It has been demonstrated that DHFR enzyme is target for longest-acting antibiotics, antiprotozoal and anti-cancerous agents (Sulfonamide, pyrimethamine, and 
methotrexate). .[32,33] Therefore, Computational studies designed to assess the pharmacology of the folate metabolic pathway lead to exploring the molecule with desire pharmacological outcomes. Repurposing of drugs is a new approach in which identification of new pharmacological use of already approved drugs for a different activity. Further, the advantages of this approach included a known complete profile of drugs, and the expenditure of drug development reduces exponentially.

\section{Conclusion}

Fungal infections are responsible for both local as well as systemic effects in the humans. The invasive fungal infection involves different organ systems including lungs and results in pneumonia like symptoms characterized by difficulty in breathing. These breathing problems are having symptomatic similarity with COVID19 infections. The COVID19 patients being immunocompromised are highly prone to get fungal infections. The increasing number of fungal infections among the COVID19 patients worsening the scenario by increasing the complications in COVID19 patients resulting in the multiorgan failure leading to the death. The existing antifungal therapy is seeming to be inefficient to counter the fungal infections among the COVID19 patients because of the development of drug resistance by the pathogen and co-infection among the COVID-19 patients. Fungal DHFR was explored as a potential antifungal drug target involved in the biosynthesis of the pathogenic nuclear material. Thus, in the current research computational repurposing approach has been employed to identify potential inhibitors of the fungal DHFR to develop a newer antifungal therapy. Benzbromarone was identified as a potent inhibitor of fungal DHFR by molecular docking simulation-based screening of a ligand library of existing approved drugs followed by the confirmation of the stability with respect to time by molecular dynamics simulations.

\section{Decleration}

Funding: No funding or financial aid have been received to support this research.

Conflicts of interest/Competing interests: Authors declares that there are no financial or non-financial interests that are directly or indirectly related to the work

Availability of data and material: Not applicable

Code availability: Not applicable

Authors' contributions: Both the authors have contributed equally for the execution of current research. The framework is designed and executed by SM while the validation of the research outcomes and drafting of the manuscript was done by AT.

\section{References}


1. DeJarnette C, Luna-Tapia A, Estredge LR, Palmer GEJM (2020) Dihydrofolate Reductase Is a Valid Target for Antifungal Development in the Human Pathogen Candida albicans. 5 (3)

2. Benedict K, Kobayashi M, Garg S, Chiller T, Jackson BR (2020) Symptoms in blastomycosis, coccidioidomycosis, and histoplasmosis versus other respiratory illnesses in commercially insured adult outpatients, United States, 2016-2017. Clin Infect Dis. doi:10.1093/cid/ciaa1554

3. Nucci M, Barreiros G, Guimarães LF, Deriquehem VA, Castiñeiras AC, Nouér SAJM (2020) Increased incidence of candidemia in a tertiary care hospital with the COVID-19 pandemic.

4. Hughes S, Troise O, Donaldson H, Mughal N, Moore LSP (2020) Bacterial and fungal coinfection among hospitalized patients with COVID-19: a retrospective cohort study in a UK secondary-care setting. Clin Microbiol Infect 26 (10):1395-1399. doi:10.1016/j.cmi.2020.06.025

5. Chen N, Zhou M, Dong X, Qu J, Gong F, Han Y, Qiu Y, Wang J, Liu Y, Wei Y, Xia J, Yu T, Zhang X, Zhang L (2020) Epidemiological and clinical characteristics of 99 cases of 2019 novel coronavirus pneumonia in Wuhan, China: a descriptive study. Lancet 395 (10223):507-513. doi:10.1016/S0140-6736(20)30211-7

6. Walsh TJ, Teppler H, Donowitz GR, Maertens JA, Baden LR, Dmoszynska A, Cornely OA, Bourque MR, Lupinacci RJ, Sable CA, dePauw BE (2004) Caspofungin versus liposomal amphotericin B for empirical antifungal therapy in patients with persistent fever and neutropenia. N Engl J Med 351 (14):1391-1402. doi:10.1056/NEJMoa040446

7. DeJarnette C, Luna-Tapia A, Estredge LR, Palmer GE (2020) Dihydrofolate Reductase Is a Valid Target for Antifungal Development in the Human Pathogen Candida albicans. mSphere 5 (3). doi:10.1128/mSphere.00374-20

8. Posteraro B, Torelli R, Vella A, Leone PM, De Angelis G, De Carolis E, Ventura G, Sanguinetti M, Fantoni M (2020) Pan-Echinocandin-Resistant Candida glabrata Bloodstream Infection Complicating COVID-19: A Fatal Case Report. J Fungi (Basel) 6 (3). doi:10.3390/jof6030163

9. Kullberg BJ, Viscoli C, Pappas PG, Vazquez J, Ostrosky-Zeichner L, Rotstein C, Sobel JD, Herbrecht R, Rahav G, Jaruratanasirikul S, Chetchotisakd P, Van Wijngaerden E, De Waele J, Lademacher C, Engelhardt M, Kovanda L, Croos-Dabrera R, Fredericks C, Thompson GR (2019) Isavuconazole Versus Caspofungin in the Treatment of Candidemia and Other Invasive Candida Infections: The ACTIVE Trial. Clin Infect Dis 68 (12):1981-1989. doi:10.1093/cid/ciy827

10. Li X, Hou Y, Yue L, Liu S, Du J, Sun S (2015) Potential Targets for Antifungal Drug Discovery Based on Growth and Virulence in Candida albicans. Antimicrob Agents Chemother 59 (10):5885-5891. doi:10.1128/AAC.00726-15

11. Mujwar S, Deshmukh R, Harwansh RK, Gupta JK, Gour A (2019) Drug Repurposing Approach for Developing Novel Therapy Against Mupirocin-Resistant Staphylococcus aureus. Assay Drug Dev Technol 
17 (7):298-309. doi:10.1089/adt.2019.944

12. Mujwar S, Kumar VJA, technologies dd (2020) Computational Drug Repurposing Approach to Identify Potential Fatty Acid-Binding Protein-4 Inhibitors to Develop Novel Antiobesity Therapy. 18 (7):318-327

13. Jain R, Mujwar SJSC (2020) Repurposing metocurine as main protease inhibitor to develop novel antiviral therapy for COVID-19. 31 (6):2487-2499

14. Shah K, Mujwar S, Gupta JK, Shrivastava SK, Mishra P (2019) Molecular Docking and In Silico Cogitation Validate Mefenamic Acid Prodrugs as Human Cyclooxygenase-2 Inhibitor. Assay Drug Dev Technol 17 (6):285-291. doi:10.1089/adt.2019.943

15. Berman HM, Westbrook J, Feng Z, Gilliland G, Bhat TN, Weissig H, Shindyalov IN, Bourne PEJNar (2000) The protein data bank. 28 (1):235-242

16. N GD, Paulsen JL, Viswanathan K, Keshipeddy S, Lombardo MN, Zhou W, Lamb KM, Sochia AE, Alverson JB, Priestley ND, Wright DL, Anderson AC (2014) Propargyl-linked antifolates are dual inhibitors of Candida albicans and Candida glabrata. J Med Chem 57 (6):2643-2656. doi:10.1021/jm401916j

17. Pettersen EF, Goddard TD, Huang CC, Couch GS, Greenblatt DM, Meng EC, Ferrin TE (2004) UCSF Chimera-a visualization system for exploratory research and analysis. J Comput Chem 25 (13):16051612. doi:10.1002/jcc.20084

18. Pradhan P, Soni NK, Chaudhary L, Mujwar S, Pardasani KJBBRA (2015) In-silico prediction of riboswitches and design of their potent inhibitors for H1N1, H2N2 and H3N2 strains of influenza virus. 12 (3):2173-2186

19. Kaushal SK, Brijendra S, Mujwar S, Prakash BS (2019) Molecular Docking based analysis to elucidate the DNA Topoisomerase Ilbeta as the potential target for the Ganoderic acid, A natural therapeutic agent in cancer therapy. Curr Comput Aided Drug Des. doi:10.2174/1573409915666190820144759

20. Kaur A, Mujwar S, Adlakha NJIJoCB, Design D (2016) In-silico analysis of riboswitch of Nocardia farcinica for design of its inhibitors and pharmacophores. 9 (3):261-276

21. Forli S, Huey R, Pique ME, Sanner MF, Goodsell DS, Olson AJ (2016) Computational protein-ligand docking and virtual drug screening with the AutoDock suite. Nat Protoc 11 (5):905-919.

doi:10.1038/nprot.2016.051

22. Mujwar S PK (2015) Prediction of riboswitch as a potential drug target and design of its optimal inhibitors for Mycobacterium tuberculosis. International Journal of Computational Biology and Drug Design 8 (4):326-347

23. Mujwar S PK (2015) Prediction of Riboswitch as a potential drug target for infectious diseases: An Insilico case study of anthrax. Journal of Medical Imaging and Health Informatics 5 (1):7-16 
24. Minaz N, Razdan R, Hammock BD, Mujwar S, Goswami SK (2019) Impact of diabetes on male sexual function in streptozotocin-induced diabetic rats: Protective role of soluble epoxide hydrolase inhibitor. Biomed Pharmacother 115:108897. doi:10.1016/j.biopha.2019.108897

25. Mujwar SJCiB, Medicine (2021) Computational Repurposing of Tamibarotene Against Triple Mutant Variant of SARS-CoV-2.104748

26. System SR-DMD (2020) Maestro-Desmond Interoperability Tools, Schrödinger, New York, NY, 2021. D. E. Shaw Research, New York, NY, 2021.

27. Al-Hatmi AM, Mohsin J, Al-Huraizi A, Khamis FJJol (2021) COVID-19 associated invasive candidiasis. 82 (2):e45-e46

28. Buehler PK, Zinkernagel AS, Hofmaenner DA, Garcia PDW, Acevedo CT, Gómez-Mejia A, Shambat SM, Andreoni F, Maibach MA, Bartussek JJCRM (2021) Bacterial pulmonary superinfections are associated with longer duration of ventilation in critically ill COVID-19 patients. 2 (4):100229

29. Kordalewska M, Guerrero KD, Garcia-Rubio R, Jiménez-Ortigosa C, Mediavilla JR, Cunningham MH, Hollis F, Hong T, Chow KF, Kreiswirth BNJJoF (2021) Antifungal Drug Susceptibility and Genetic Characterization of Fungi Recovered from COVID-19 Patients. 7 (7):552

30. Verweij PE, Gangneux J-P, Bassetti M, Brüggemann RJ, Cornely OA, Koehler P, Lass-Flörl C, van de Veerdonk FL, Chakrabarti A, Hoenigl MJTLM (2020) Diagnosing COVID-19-associated pulmonary aspergillosis. 1 (2):e53-e55

31. Shah B, Modi P, Sagar SRJLs (2020) In silico studies on therapeutic agents for COVID-19: Drug repurposing approach. 252:117652

32. Chen N, Zhou M, Dong X, Qu J, Gong F, Han Y, Qiu Y, Wang J, Liu Y, Wei YJTI (2020) Epidemiological and clinical characteristics of 99 cases of 2019 novel coronavirus pneumonia in Wuhan, China: a descriptive study. 395 (10223):507-513

33. White L, Dhillon R, Cordey A, Hughes H, Faggian F, Soni S, Pandey M, Whitaker H, May A, Morgan M (2020) A national strategy to diagnose COVID-19 associated invasive fungal disease in the ICU.

\section{Figures}




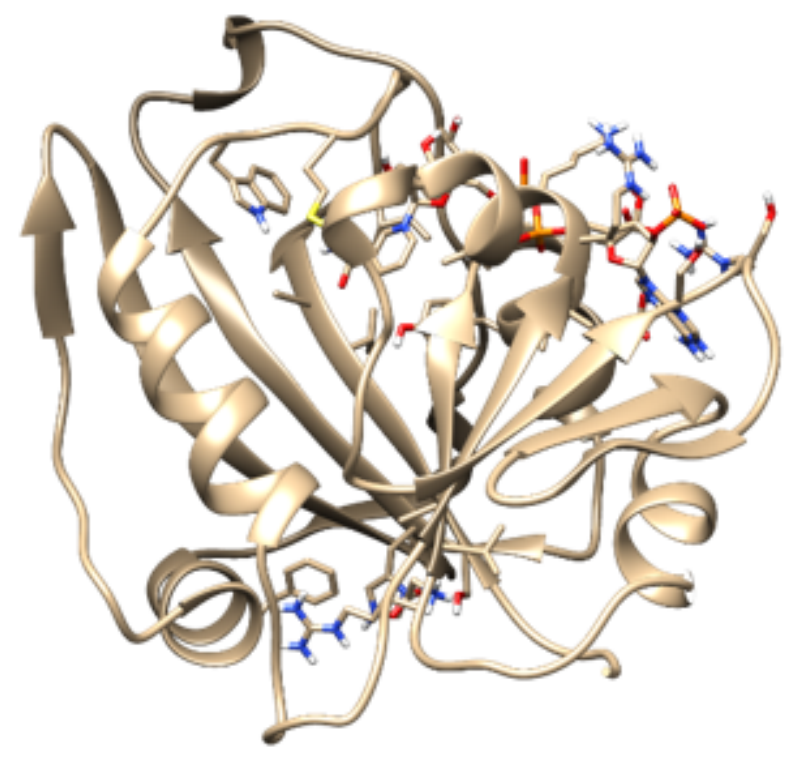

Figure 1

Monomeric processed macromolecular structure of fungal DHFR procured from the RCSB database protein data bank. 


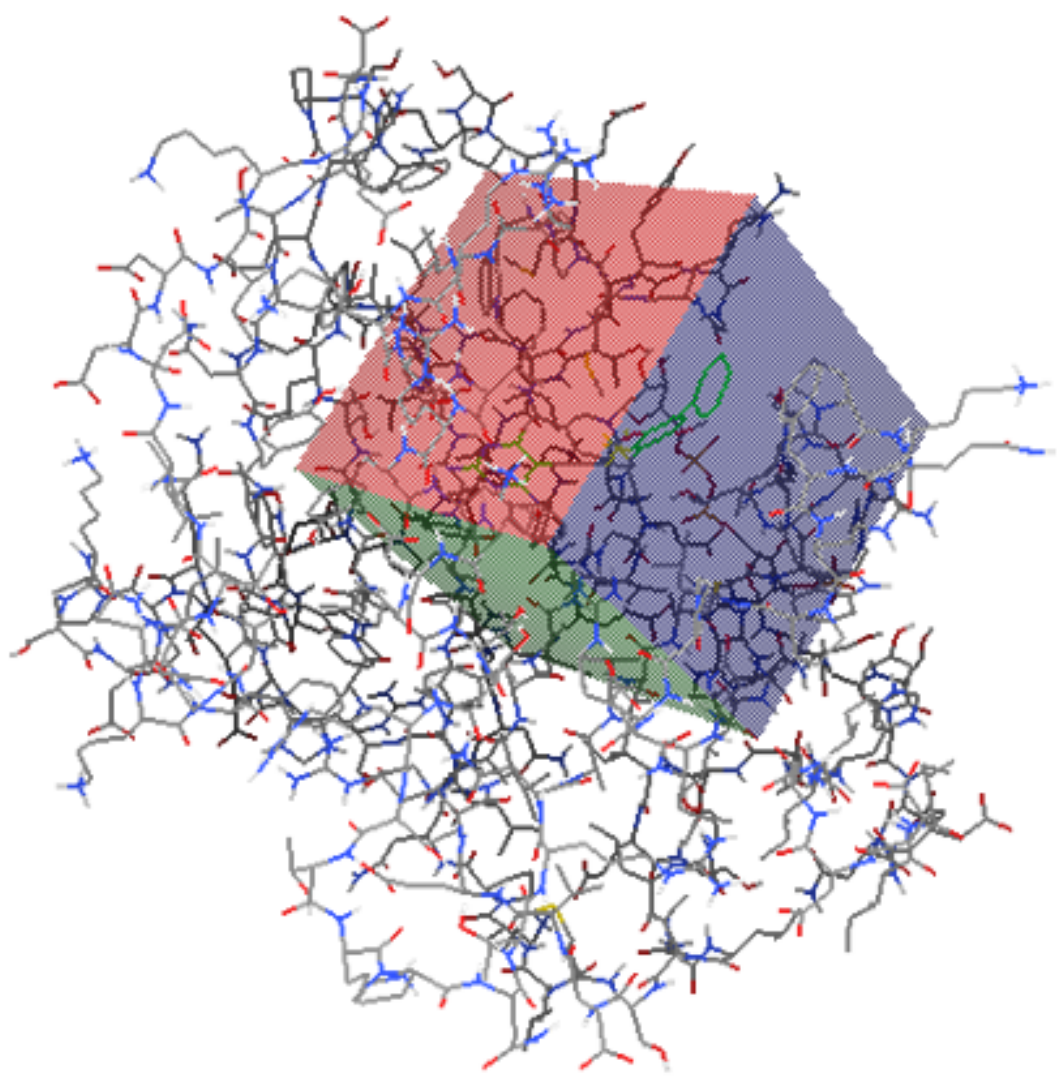

Figure 2

Three-dimensional imaginary grid-box was prepared by wrapping all the macromolecular binding residues interacting with the ligand. 


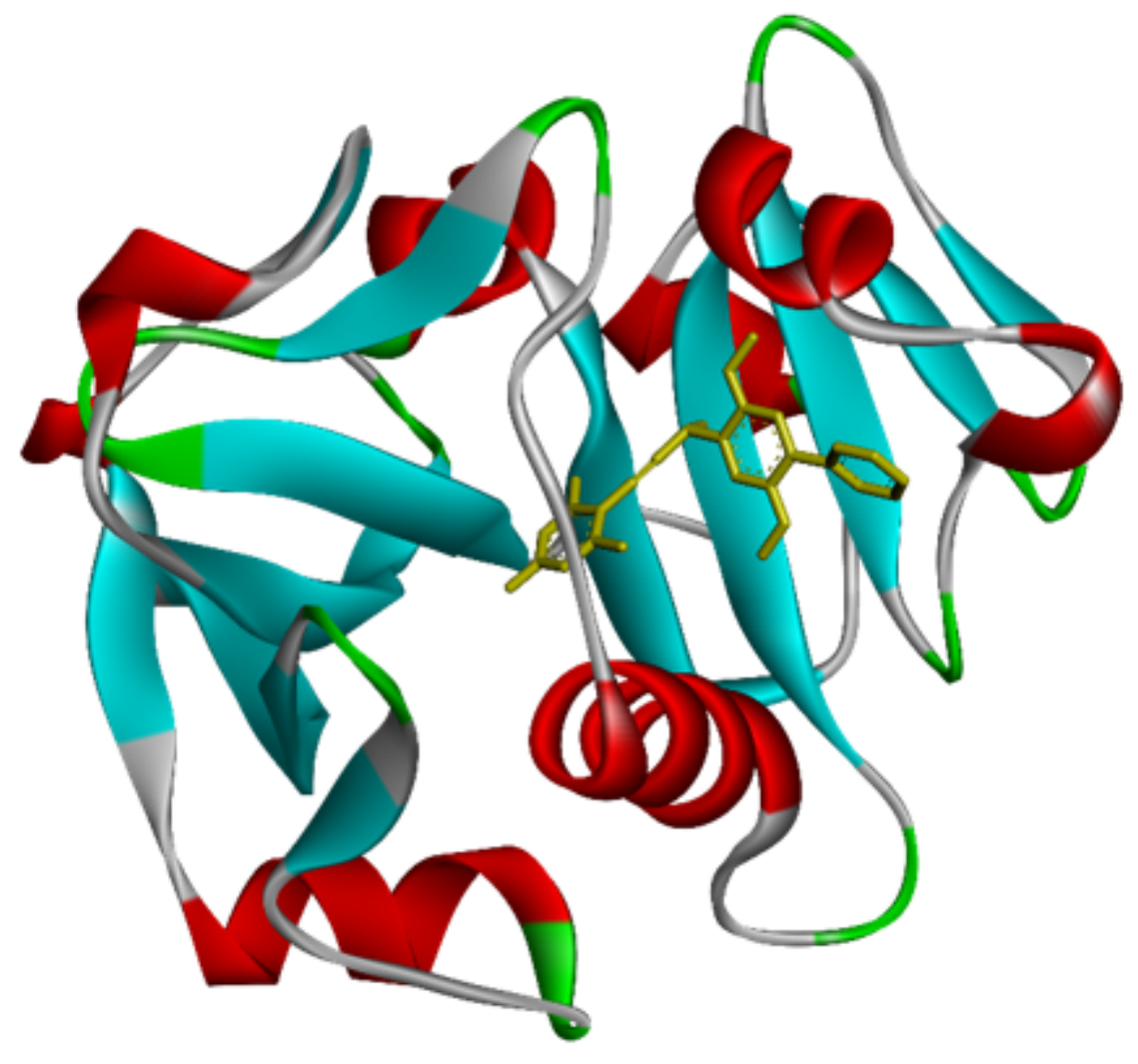

Figure 3

The three-dimensional binding confirmation ligand $18 \mathrm{G}$ against the target receptor fungal DHFR.

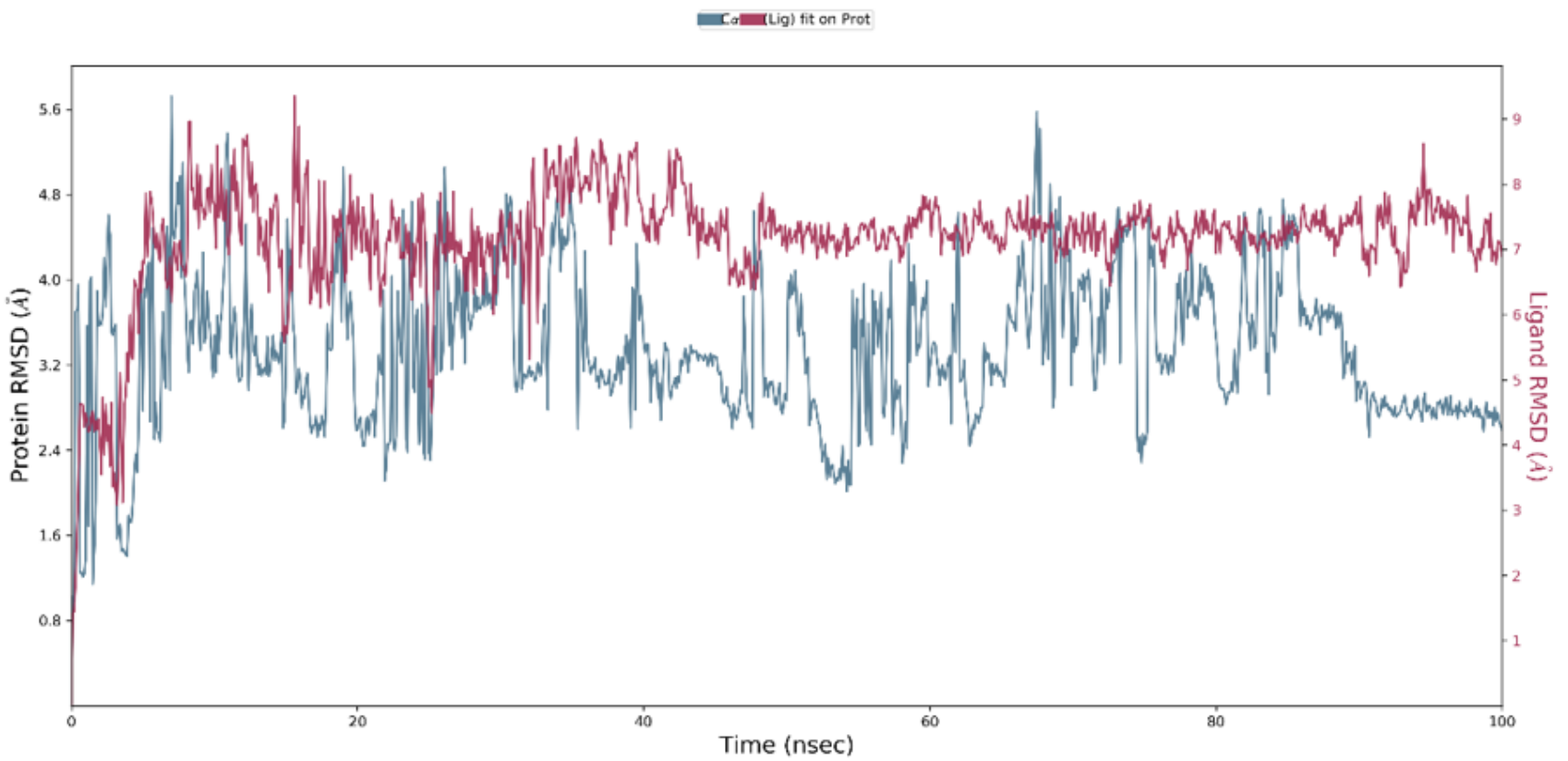

Figure 4 
RMSD trajectory for the macromolecular target as well as ligand obtained after performing molecular dynamics simulation.

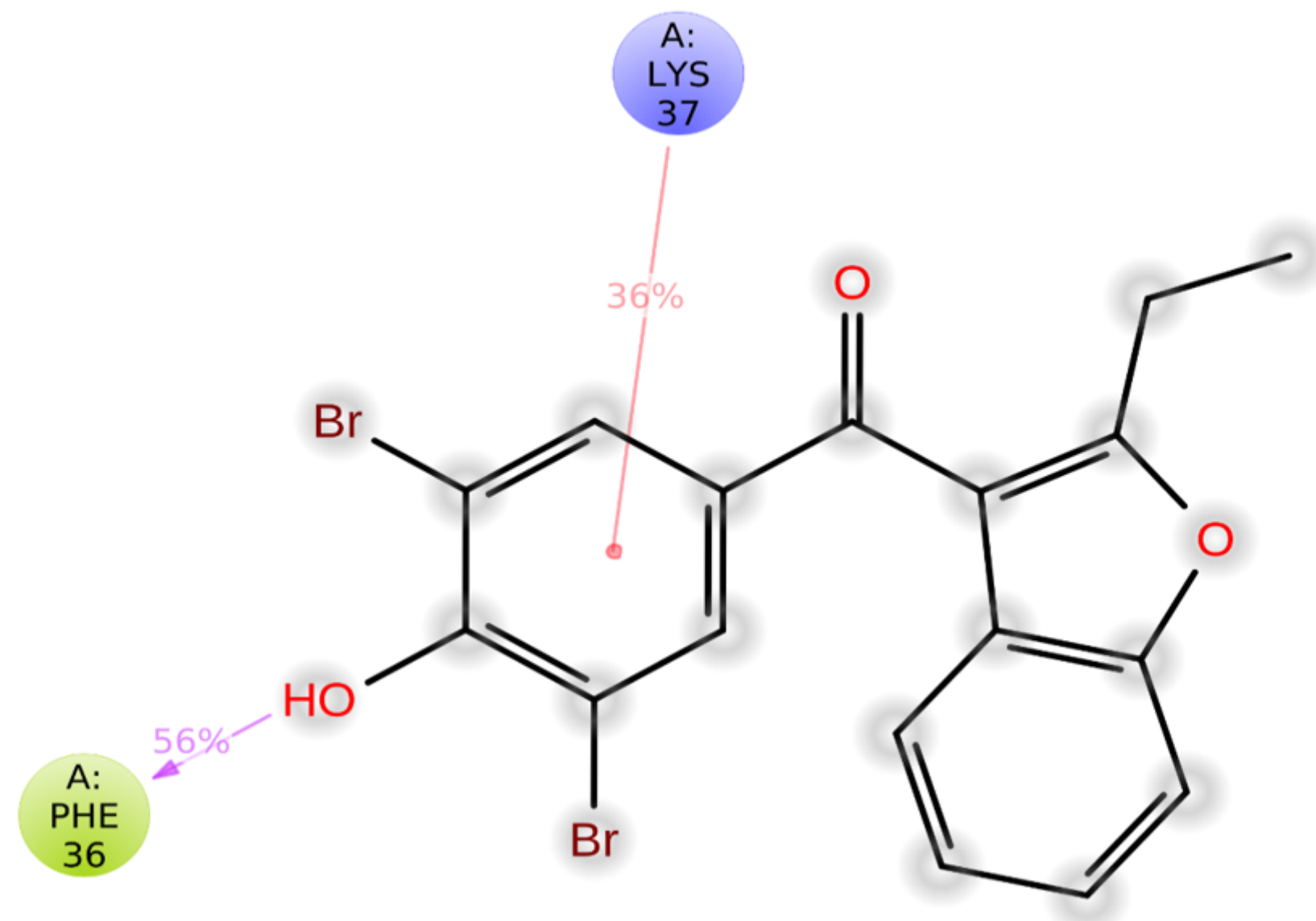

Figure 5

Interaction analysis of Benzbromarone against fungal DHFR.

\section{Supplementary Files}

This is a list of supplementary files associated with this preprint. Click to download.

- desmondmdjob1outpl1.pdf 